Ahmet Usta, Emin Gemcioglu*, Salih Baser, Osman Ersoy, Yunus Halil Polat, Abdülsamet Erden and Seval Izdes

\title{
Comparison of APACHE II scores and mortality with CRP/albumin, neutrophil/lymphocyte and thrombocyte/lymphocyte ratios in patients admitted to internal medicine and anesthesia reanimation intensive care unit
}

https://doi.org/10.1515/labmed-2021-0061

Received May 22, 2021; accepted September 24, 2021; published online November 9, 2021

\section{Abstract}

Objectives: This study aimed to evaluate the relationship between C-reactive protein/albumin (CRP/Alb), neutrophil/lymphocyte (NLR), platelet/lymphocyte (PLR) ratios and the Acute Physiology And Chronic Health Evaluation II (APACHE II) score and 28-day mortality among 400 patients admitted to internal medicine and anesthesia reanimation intensive care unit (ICU).

Methods: This prospective study included a total of 400 patients who were admitted to hospital internal medicine and anesthesia reanimation ICUs.

*Corresponding author: Emin Gemcioglu, MD, Department of Internal Medicine, Ankara City Hospital, Ankara 06100, Turkey, Phone: +90 505 9369141, Fax: +90 312 3100194,

E-mail: egemcioglu@gmail.com.

https://orcid.org/0000-0001-9751-8452

Ahmet Usta, Department of Internal Medicine, Yıldırım Beyazıt University School of Medicine, Ankara, Turkey,

E-mail: ahmet.2254@hotmail.com. https://orcid.org/0000-00033149-9505

Salih Baser, Department of Internal Medicine, Yıldırım Beyazıt University Ankara City Hospital, Ankara, Turkey,

E-mail: drsalihbaser@gmail.com. https://orcid.org/0000-0002-

3448-6454

Osman Ersoy and Yunus Halil Polat, Department of Gastroenterology, Yıldırım Beyazıt University School of Medicine, Ankara, Turkey, E-mail: oersoy@yahoo.com.tr (0. Ersoy), yunushpolat@gmail.com (Y.H. Polat). https://orcid.org/0000-0002-1364-5962 (0. Ersoy). https://orcid.org/0000-0002-2388-5388 (Y.H. Polat)

Abdülsamet Erden, Department of Rheumatology, Yıldırım Beyazıt University School of Medicine, Ankara, Turkey.

https://orcid.org/0000-0002-8084-2018

Seval Izdes, Department of Intensive Care, Yıldırım Beyazıt University School of Medicine, Ankara, Turkey, E-mail: sevalizdes@yahoo.com. https://orcid.org/0000-0001-9856-2391
Results: The most common reasons for ICU admission were pneumonia (29.3\%), gastrointestinal bleeding (10.3\%), acute exacerbation of chronic kidney disease (CKD) (10.3\%), and acute kidney injury (7.5\%). The comparison of the laboratory findings with survival outcomes revealed that among the patients with acute exacerbation of CKD, the median NLR ( $\mathrm{p}=0.043)$ and median CRP/Alb ( $\mathrm{p}=0.021)$ were significantly higher in patients who died. For all of the patients, the APACHE II score was positively correlated with CRP ( $p<0.001)$ and CRP/Alb ( $<<0.001)$, negatively correlated with Alb $(p<0.001)$, positively correlated with the NLR $(\mathrm{p}<0.001)$, and positively correlated with the PLR.

Conclusions: The APACHE II score was significantly correlated with the CRP/Alb ratio, NLR, and PLR. The NLR and CRP/Alb ratio were statistically associated with mortality in patients hospitalized for acute exacerbation of CKD.

Keywords: APACHE II; CRP/albumin ratio; intensive care; mortality; neutrophil/lymphocyte ratio; thrombocyte/ lymphocyte ratio.

\section{Introduction}

Intensive care scoring systems are widely used to predict recovery, determine the severity of the disease and the degree of organ dysfunction, evaluate treatment effectiveness, standardize patients in clinical trials, and compare the performances of intensive care units (ICUs). The Acute Physiology and Chronic Health Examination (APACHE) II score was originally developed for critically ill patients in intensive care units (ICUs). Patient data obtained from daily measurements are used in scoring, and many clinical scoring systems have been defined as follows: It consists of two main parts: "prognostic" scoring systems that predict mortality and "organ failure" scoring systems that evaluate morbidity. APACHE II. It consists of three parts: acute physiology score, age and chronic health assessment 
(Table 1). The results from these three sections are summed and patient mortality is determined. The values recorded in APACHE II are the values that deviate the most from normal in the first $24 \mathrm{~h}$ of the patient's intensive care unit. Chronological age was added as a weighted score, since it reflects the decrease in physiological reserve and is an important factor that determines the risk of mortality regardless of disease severity in case of acute illness. The APACHE II scoring system also has its inadequacies: Elderly patients can score higher than necessary, and acute physiology score does not have regulated measures for use of drugs for hemodynamic support therapy, mechanical ventilation $[1,2]$. Accordingly, alternative scoring systems are needed for patient assessment in the ICU.

$\mathrm{C}$-reactive protein (CRP) is a positive acute-phase reactant that is synthesized by the liver, and its serum levels increase within hours in response to inflammation and infection [3]. CRP exerts both pro and anti-inflammatory properties. In addition to being an indicator of infection and inflammation, it has a wide range of biological properties and functions [4]. Albumin (Alb) is a negative acute-phase reactant that is synthesized by the liver, and its serum levels decrease during inflammation. Serum Alb acts as an important modulator of plasma oncotic pressure and a carrier of endogenous and exogenous (i.e., drugs) compounds [5]. The CRP/Alb ratio is a novel inflammatory prognostic parameter that is associated with inflammation severity and mortality. It is a more accurate indicator of inflammatory status when compared to CRP or Alb alone. Recent studies have investigated the parameters derived from complete blood count ( $\mathrm{CBC}$ ) results, such as the neutrophil/lymphocyte ratio (NLR) and platelet/lymphocyte ratio (PLR), as novel indicators of inflammation in the context of many diseases [6]. The physiologic response of leukocytes to stress can result in an increase in neutrophil counts and decline in lymphocyte counts, and the ratio of these neutrophil and lymphocyte counts is used as an indicator of inflammation. Neutrophil activation releases reactive oxygen species, cytokines, proteases, and cationic proteins (such as elastase and lactoferrin), and these neutrophil activation products have been associated with the severity of numerous inflammatory diseases [7]. Platelets also interact with endothelial cells, leukocytes (monocytes, neutrophils, dendritic cells, T-cells) and progenitor cells, and cause the migration of inflammatory cells to lesions, the release of large amounts of inflammatory cytokines, and ultimately, the formation of an inflammatory zone [8].

This study aimed to evaluate the relationship between the CRP/Alb ratio, NLR, and PLR, and the APACHE II score and 28-day mortality among 400 patients admitted to the ICU for various reasons.

\section{Materials and methods}

This prospective study included a total of 400 patients admitted to the hospital internal medicine and anesthesia reanimation ICUs between September 2019 and March 2020. The age, gender, reason for ICU admission, comorbidities, sepsis, white blood cell (WBC) count, hemoglobin, thrombocyte, neutrophil, and lymphocyte counts, red cell distribution width (RDW), CRP, Alb, procalcitonin, antibiotic use, 28-day mortality, and APACHE II scores of the patients during hospitalization were recorded. The association between the admission APACHE II scores and 28-day mortality, and biochemistry (CRP and $\mathrm{Alb}$ ) and CBC parameters (thrombocyte, neutrophil, lymphocyte counts) measured within $24 \mathrm{~h}$ after ICU admission were analyzed. Demographic characteristics, clinical and laboratory results, treatment, and outcome were recorded using a standard case report form.

The inclusion criteria were being $\geq 18$ years of age and being admitted to the internal medicine and anesthesia reanimation ICUs, while the exclusion criteria were trauma, malignancies, chronic hepatitis and cirrhosis of the liver, and malnutrition due to potentially altering CRP and Alb levels. Also excluded were patients receiving exogenous Alb therapy.

For this study, the CRP was measured using a commercial kit in a Siemens BN II System autoanalyzer (Munich, Germany) based on nephelometry. Alb was measured with the bromocresol green colorimetric method using a commercial kit on a Roche Cobas 8000 automated platform (Mannheim, Germany).

Three inflammatory markers were used to determine the association between APACHE II and mortality among ICU patients, comprising the $\mathrm{CRP} / \mathrm{Alb}$ ratio, neutrophil to lymphocyte ratio (NLR), and platelet to lymphocyte ratio (PLR).

CBC was performed using blood samples collected into K2EDTA tubes in an Sysmex XE-2100 automated CBC device (Mundelein, IL, USA). This study was approved by the local Ethics Committee (approval number 26379996/106, date 18/09/2019).

\section{Statistical analysis}

Data were analyzed using IBM SPSS Statistics for Windows 20.0 (IBM Corp., Armonk, NY, USA). Normality of data distribution was evaluated using the Kolmogorov-Smirnov test. Normally distributed data were expressed as the mean \pm standard deviation, and non-normally distributed data were expressed as the median (minimum-maximum). Categorical variables were expressed as numbers and percentages. Pairwise comparison was performed using the student $t$-test and Mann-Whitney U test for normally and non-normally distributed numerical variables, respectively. The chi-square and Fisher exact tests were used for the comparison of categorical variables. Correlations between numerical variables were evaluated using the Spearman correlation analysis. A p-value $<0.05$ was accepted as statistically significant.

\section{Results}

The study included a total of 400 patients, comprising 203 females (50.8\%) and 197 males (49.2\%). The mean age of the patients was $73.2 \pm 16.1$ years (range 18-103). 


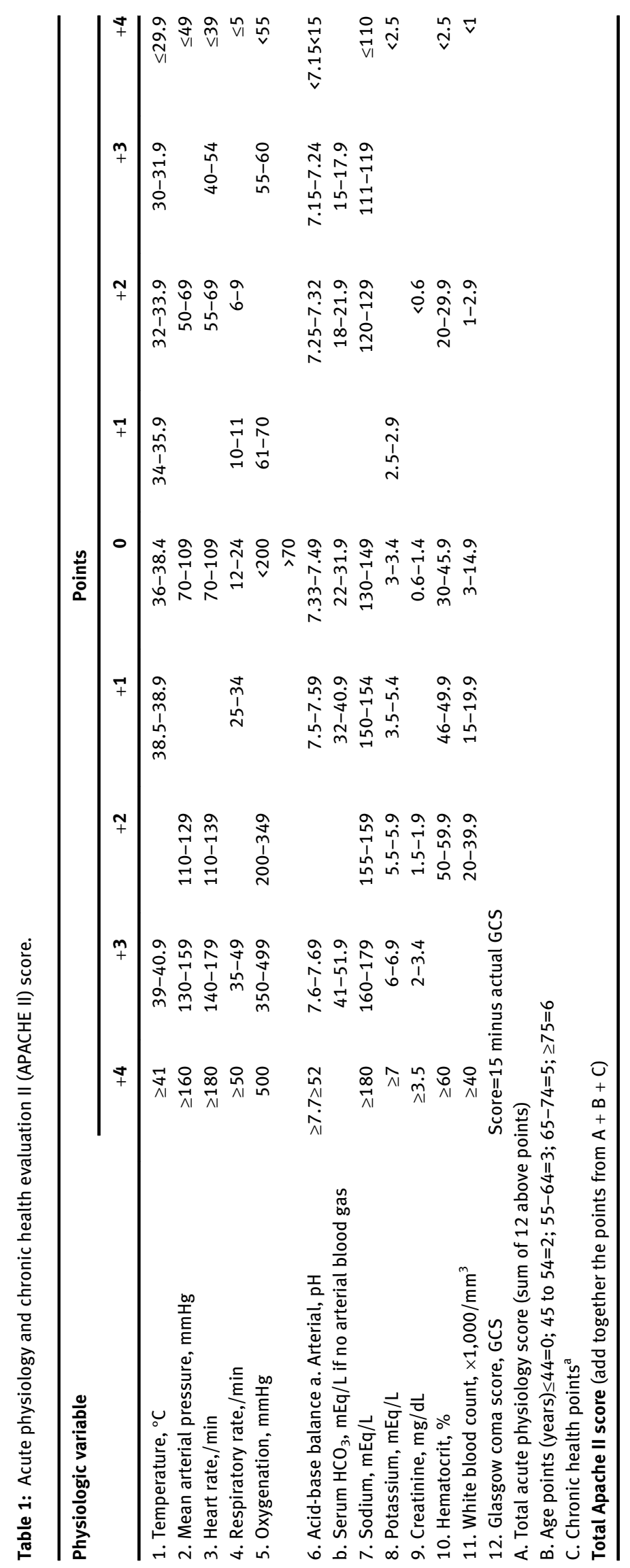


Of the patients, 95.5\% $(\mathrm{n}=382)$ had at least one comorbidity, the most common being hypertension $(50.5 \%$, $\mathrm{n}=202)$, diabetes mellitus (34.8\%, $\mathrm{n}=139)$, chronic kidney disease (CKD) $(283 \%, \mathrm{n}=139)$, coronary artery disease (24.5\%, $\mathrm{n}=98)$, and chronic heart failure $(24 \%, \mathrm{n}=96)$ (Figure 1). The most common reasons for ICU admission were pneumonia (29.3\%), gastrointestinal (GI) bleeding (10.3\%), acute exacerbation of CKD (10.3\%), acute kidney injury (7.5\%), urosepsis (5.5\%), decompensated heart failure (4.3\%), hypernatremia (4.3\%), and diabetic ketoacidosis (3.3\%). Of the patients admitted to the ICU, 37.3\% $(\mathrm{n}=149)$ had sepsis. The 28-day mortality rate was $35.5 \%$ $(n=142)$ (Table 2). The distribution of laboratory results according to survival among these patients is presented in Table 3.

Accordingly, among the patients with acute exacerbation of CKD, the neutrophil count (median 16,630 vs. 10,780, $\mathrm{p}=0.030$ ), WBC (median 14,000 vs. $8,820, \mathrm{p}=0.025$ ), NLR (median 11.8 vs. 5.7, $\mathrm{p}=0.043$ ), and CRP/Alb (median 38 vs. $11.1, \mathrm{p}=0.021$ ) were significantly higher in patients who died when compared to those who survived (Figure 2).

For all of the patients, the APACHE II scores were strongly positively correlated with the RDW $(r=0.712$, $\mathrm{p}<0.001)$, CRP $(\mathrm{r}=0.840, \mathrm{p}<0.001)$, and CRP/Alb $(\mathrm{r}=0.852$, $\mathrm{p}<0.001)$, strongly negatively correlated with Alb $(\mathrm{r}=-0.756$, $\mathrm{p}<0.001)$ and moderately positively correlated with procalcitonin $(r=0.541, p<0.001)$ and the NLR $(r=0.559, p<0.001)$ (Table 4) (Figure 3). Moreover, the APACHE II scores were significantly weakly positively correlated with the WBC, neutrophil, AST, creatinine, urea, potassium, and PLR, and significantly weakly negatively correlated with hemoglobin, lymphocyte count, and calcium. Similar correlations were observed when the variables were analyzed in terms of 28-day survival (Table 4).
Table 2: Reasons for ICU admission and ICU sepsis incidence and survival outcomes.

\begin{tabular}{ll}
\hline Reason for ICU admission & Total $\mathbf{n}=\mathbf{4 0 0} \mathbf{n}(\%)$ \\
\hline Pneumonia & $117(29.3)$ \\
Gastrointestinal bleeding & $41(10.3)$ \\
Acute exacerbation of CKD & $41(10.3)$ \\
Acute kidney disease & $30(7.5)$ \\
Urosepsis & $22(5.5)$ \\
Decompensated heart failure & $17(4.3)$ \\
Hypernatremia & $17(4.3)$ \\
Diabetic ketoacidosis & $13(3.3)$ \\
\hline Events and survival & Total $\mathbf{n}=400 \mathrm{n}(\%)$ \\
\hline Sepsis & $149(37.3)$ \\
28-day survival & \\
Survived & $258(64.5)$ \\
Died & $142(35.5)$ \\
\hline
\end{tabular}

\section{Discussion}

Here in, the most common reasons for ICU admission were pneumonia, GI bleeding, acute exacerbation of CKD, and AKI. Survival was not found to be significantly correlated with the CRP/Alb ratio, median NLR, or PLR among the patients with pneumonia, GI bleeding, or AKI; however, survival was significantly associated with the median neutrophil count, median WBC, median NLR, and median CRP/Alb among the patients with an acute exacerbation of CKD. When selecting a predictive scoring system, one must consider the feasibility, ease of use, applicability, and performance of the system in the relevant population. The ratios that were included in the current study were easy to use. Therefore, these ratios were compared with mortality and the APACHE II score, the reliability of which has been

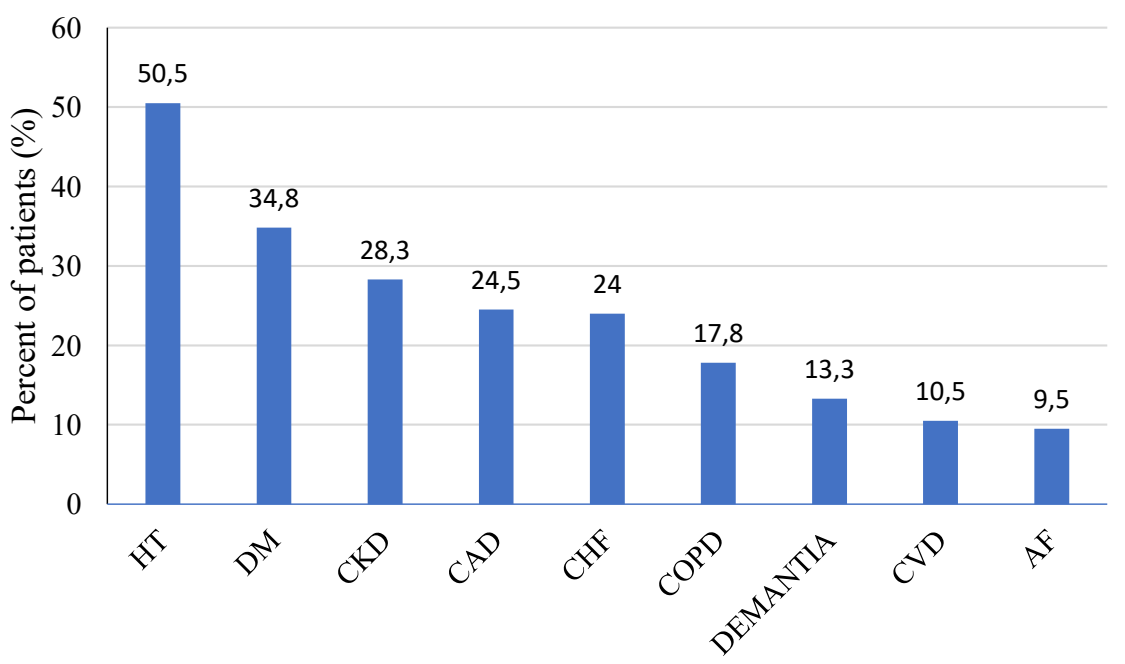

Figure 1: Most common comorbidities. $\mathrm{HT}$, hypertension; DM, diabetes mellitus; CKD, chronic kidney disease; CAD, coronary artery disease; $\mathrm{CHF}$, chronic heart failure; COPD, chronic obstructive pulmoner disease; $A F$, atrial fibrillation. 


\begin{tabular}{|c|c|c|c|c|c|c|c|}
\hline$\frac{9}{5}$ & 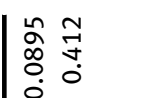 & $\begin{array}{ccc}n & \substack{n \\
\hat{\alpha}} \\
0 & 0 \\
0 & 0 \\
0\end{array}$ & $\stackrel{\substack{+m}}{m}$ & $\begin{array}{l}n \\
m \\
\dot{m}\end{array}$ & 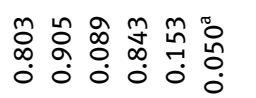 & i & $\begin{array}{l}\stackrel{\pi}{\tilde{N}} \\
\stackrel{0}{0}\end{array}$ \\
\hline 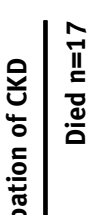 & 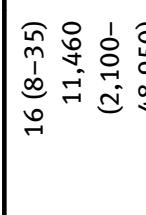 & 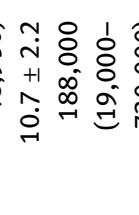 & 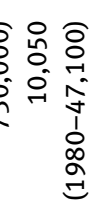 & 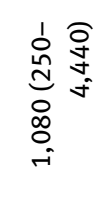 & 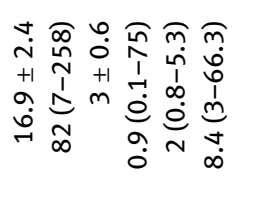 & 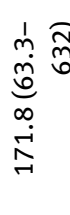 & S. \\
\hline 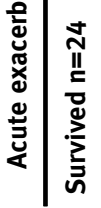 & 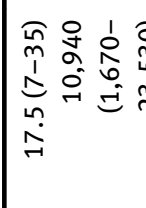 & 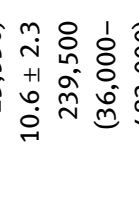 & 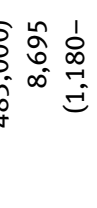 & 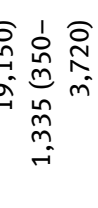 & 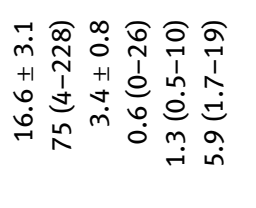 & 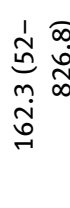 & 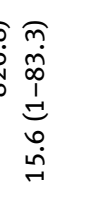 \\
\hline$\frac{9}{\frac{9}{n ! n}}$ & $\begin{array}{ll}n & n \\
\infty & n \\
0 & n \\
0 & 0 \\
0\end{array}$ & 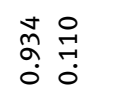 & 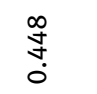 & $\begin{array}{l}2 \\
\stackrel{1}{a} \\
0\end{array}$ & 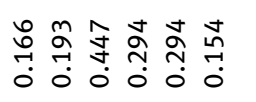 & 莗 & $\begin{array}{l}8 \\
\vdots \\
\vdots \\
0\end{array}$ \\
\hline 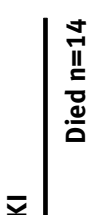 & 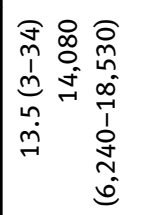 & 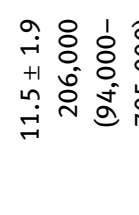 & 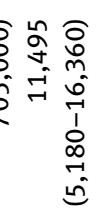 & 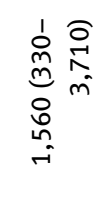 & 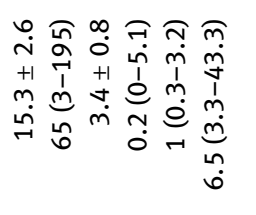 & 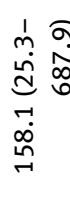 & 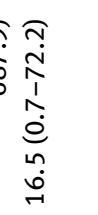 \\
\hline 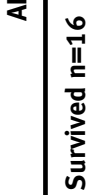 & 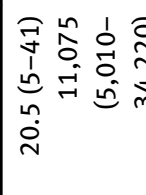 & 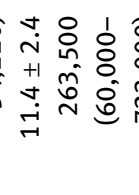 & 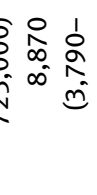 & 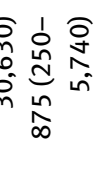 & 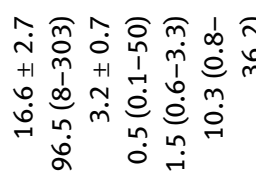 & 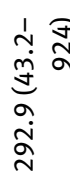 & 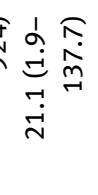 \\
\hline$\stackrel{\frac{0}{3}}{\frac{9}{5}}$ & \begin{tabular}{ll}
\multirow{2}{*}{} & $\vec{n}$ \\
\multirow{2}{*}{} & $\infty$ \\
0 & 0 \\
0
\end{tabular} & $\begin{array}{l}\hat{n} \\
\stackrel{0}{0} \\
0 \\
0\end{array}$ & $\begin{array}{l}\overrightarrow{1} \\
\infty \\
0 \\
0\end{array}$ & ริ & 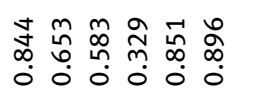 & 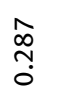 & $\begin{array}{l}n \\
\vdots \\
0\end{array}$ \\
\hline 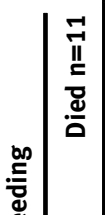 & 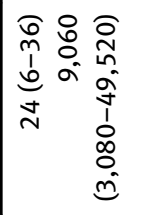 & 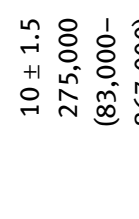 & 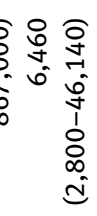 & 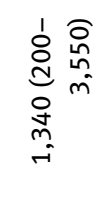 & 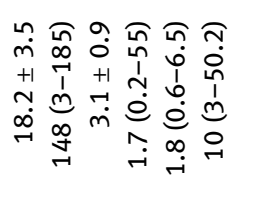 & 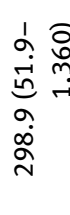 & 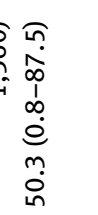 \\
\hline 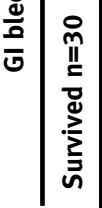 & 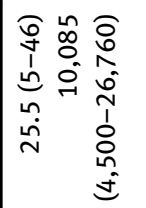 & 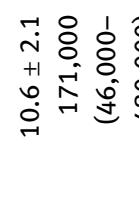 & 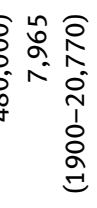 & 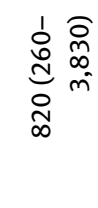 & 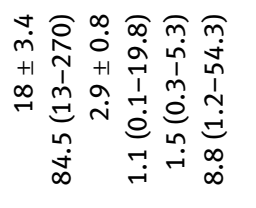 & 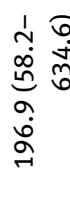 & 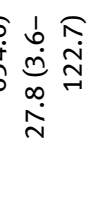 \\
\hline 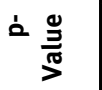 & $\stackrel{\substack{\hat{j} \\
0}}{0}$ & 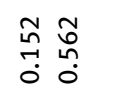 & 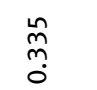 & $\underset{\substack{\hat{N} \\
0}}{a}$ & 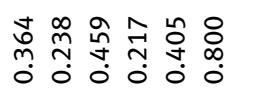 & 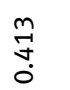 & 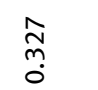 \\
\hline $\begin{array}{l}\vec{n} \\
\stackrel{-1}{2} \\
\frac{\pi}{8}\end{array}$ & 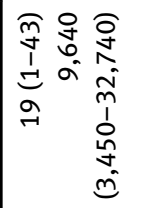 & 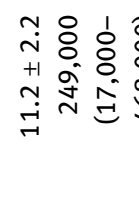 & 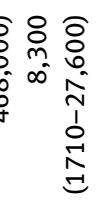 & 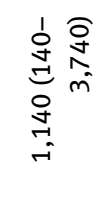 & 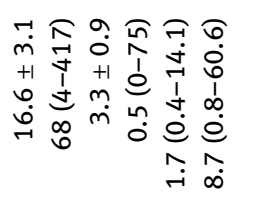 & 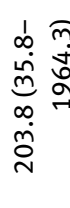 & 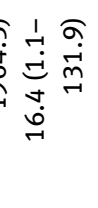 \\
\hline 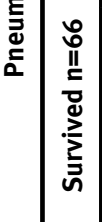 & 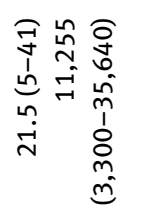 & 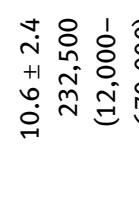 & 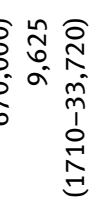 & 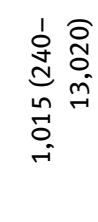 & 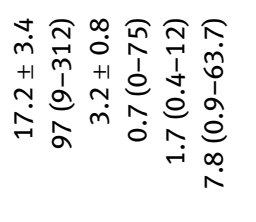 & 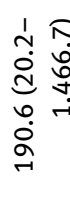 & 䇉 \\
\hline & ب & 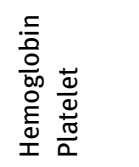 & 言 & $\begin{array}{l}\text { 芯 } \\
\text { o } \\
\text { 흘 }\end{array}$ & 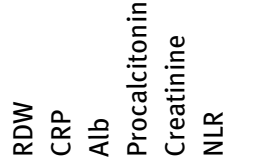 & & $\overline{\underline{k}}$ \\
\hline
\end{tabular}




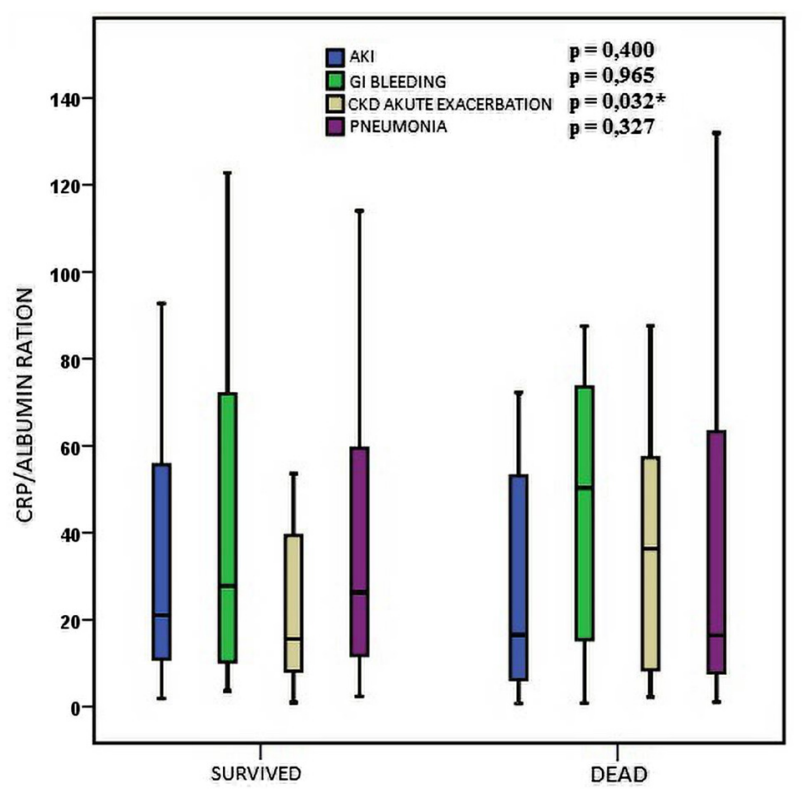

Figure 2: CRP/Alb distribution according to survival among patients admitted to the ICU for the four most common reasons.

confirmed by previous studies [9]. The prospective nature of this study allowed for the use the APACHE II scores obtained at the time of ICU admission.
The prospective nature and large sample size also made it possible to perform subgroup analyses. Although there are studies that have investigated CRP/Alb ratio, NLR, and PLR, some of which were among ICU patients, to the best of our knowledge, no studies to date have performed detailed subgroup analyses. In the current study, CKD was more prevalent among patients who died when compared to those who survived ( $38 \%$ vs. $22.9 \%, \mathrm{p}=0.001$ ). Renal insufficiency is prominent in ICU patients and usually results in poor outcomes, is commonly diagnosed late despite advances in diagnostics, and is still not adequately managed. Advances in novel diagnostic methods may improve treatment outcomes. Therefore, further studies are needed to determine the reasons behind poor prognosis and determine how to reduce acute and long-term risk among high-risk patients.

A growing number of recent studies have focused on the relationship between the NLR and PLR, and tumor characteristics. High PLR was associated with increased lymph node metastasis in colorectal and cervical cancer [10]. Xue et al. reported that increased PLR predicted a poor survival outcome among hepatocellular carcinoma patients receiving transarterial chemoembolization [11]. In their study on hepatocellular carcinoma (HCC), Zheng et al. demonstrated that the NLR and PLR were independent predictors of HCC

Table 4: Laboratory findings according to the APACHE II score.

\begin{tabular}{|c|c|c|c|c|c|c|}
\hline \multirow[t]{3}{*}{ Laboratory results } & \multicolumn{6}{|c|}{ APACHE II score } \\
\hline & \multicolumn{2}{|c|}{ Overall } & \multicolumn{2}{|c|}{ Survived } & \multicolumn{2}{|c|}{ Died } \\
\hline & $r$ & p-Value & $\mathbf{r}$ & p-Value & $r$ & p-Value \\
\hline WBC & 0.242 & $<0.001^{\mathrm{a}}$ & 0.216 & $<0.001^{\mathrm{a}}$ & 0.299 & $<0.001^{\mathrm{a}}$ \\
\hline Hemoglobin & -0.207 & $<0.001^{\mathrm{a}}$ & -0.177 & $0.004^{a}$ & -0.243 & $0.004^{\mathrm{a}}$ \\
\hline Platelet & -0.039 & 0.437 & -0.065 & 0.297 & 0.009 & 0.917 \\
\hline Neutrophil & 0.308 & $<0.001^{\mathrm{a}}$ & 0.280 & $<0.001^{\mathrm{a}}$ & 0.367 & $<0.001^{\mathrm{a}}$ \\
\hline Lymphocyte & -0.447 & $<0.001^{\mathrm{a}}$ & -0.396 & $<0.001^{\mathrm{a}}$ & -0.532 & $<0.001^{\mathrm{a}}$ \\
\hline RDW & 0.712 & $<0.001^{\mathrm{a}}$ & 0.724 & $<0.001^{\mathrm{a}}$ & 0.685 & $<0.001^{\mathrm{a}}$ \\
\hline CRP & 0.840 & $<0.001^{\mathrm{a}}$ & 0.835 & $<0.001^{\mathrm{a}}$ & 0.850 & $<0.001^{\mathrm{a}}$ \\
\hline Alb & -0.756 & $<0.001^{\mathrm{a}}$ & -0.790 & $<0.001^{\mathrm{a}}$ & -0.701 & $<0.001^{\mathrm{a}}$ \\
\hline Procalcitonin & 0.541 & $<0.001^{\mathrm{a}}$ & 0.528 & $<0.001^{a}$ & 0.571 & $<0.001^{\mathrm{a}}$ \\
\hline ALT & 0.065 & 0.192 & 0.104 & 0.096 & -0.002 & 0.978 \\
\hline AST & 0.171 & $0.001^{\mathrm{a}}$ & 0.185 & $0.003^{a}$ & 0.126 & 0.134 \\
\hline Creatinine & 0.333 & $<0.001^{\mathrm{a}}$ & 0.332 & $<0.001^{\mathrm{a}}$ & 0.347 & $<0.001^{\mathrm{a}}$ \\
\hline Urea & 0.402 & $<0.001^{\mathrm{a}}$ & 0.374 & $<0.001^{\mathrm{a}}$ & 0.453 & $<0.001^{\mathrm{a}}$ \\
\hline Sodium & 0.074 & 0.141 & 0.035 & 0.576 & 0.144 & 0.087 \\
\hline Potassium & 0.117 & $0.019^{\mathrm{a}}$ & 0.142 & $0.022^{\mathrm{a}}$ & 0.090 & 0.285 \\
\hline Calcium & -0.152 & $0.002^{\mathrm{a}}$ & -0.148 & $0.018^{\mathrm{a}}$ & -0.163 & $0.050^{\mathrm{a}}$ \\
\hline NLR & 0.559 & $<0.001^{a}$ & 0.498 & $<0.001^{\mathrm{a}}$ & 0.652 & $<0.001^{\mathrm{a}}$ \\
\hline PLR & 0.333 & $<0.001^{\mathrm{a}}$ & 0.269 & $<0.001^{a}$ & 0.438 & $<0.001^{\mathrm{a}}$ \\
\hline CRP/Alb & 0.852 & $<0.001^{\mathrm{a}}$ & 0.842 & $<0.001^{\mathrm{a}}$ & 0.867 & $<0.001^{\mathrm{a}}$ \\
\hline
\end{tabular}

${ }^{\mathrm{a}}$ Statistically significant. 

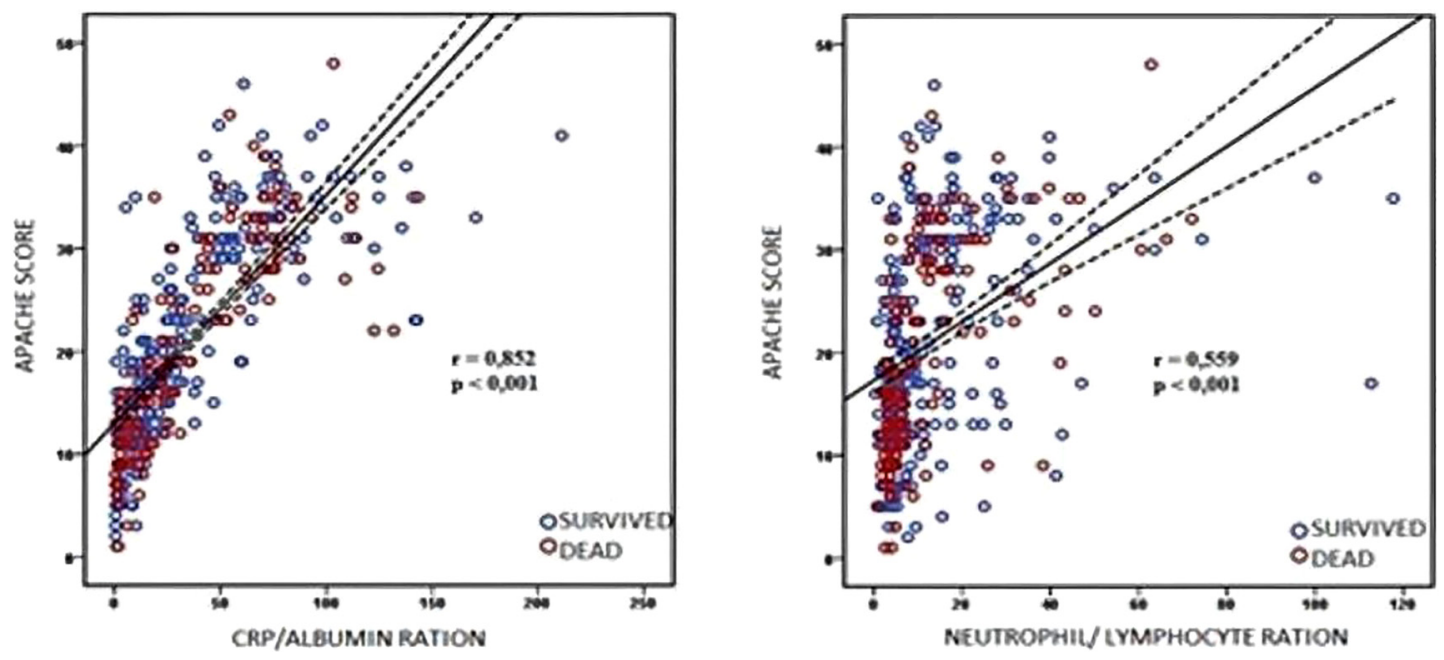

Figure 3: Indices associated with APACHE II.

recurrence and survival, and proposed that routine NLR and PLR measurements were easy-to-use and accessible parameters that should be considered as biomarkers in the clinical management of HCC [12]. In their studies on acute pulmonary embolism mortality, Soylu et al. found that an increased NLR was associated with mortality in patients with acute pulmonary embolism [13]. The reason for the superiority of NLR and PLR can be explained as follows: from an inverse perspective, the elevated ratios in the present patient group were primarily ascribed to lymphopenia. Currently, the NLR is accepted as a parameter that reflects the negative effects of high neutrophil (indicating acute inflammation) and low lymphocyte numbers (indicating physiological stress) together $[14,15]$.

The current study diverged from the literature in that survival was not found to be significantly correlated with the NLR or PLR among the patients with pneumonia, GI bleeding, or AKI; however, survival was significantly associated with the median NLR (11.8 vs. 5.7, $\mathrm{p}=0.043$ ) among the patients with an acute exacerbation of CKD. This study also included a subgroup analysis. For all of the patients, the APACHE II scores were moderately positively correlated with the NLR $(\mathrm{r}=0.559, \mathrm{p}<0.001)$. Velissaris et al. similarly found that the APACHE II scores were positively correlated with the NLR among 50 patients hospitalized for sepsis $(r=0.384, p=0.006)$ [16]. There are no other studies in the literature examining the relationship between the APACHE II scores and the NLR in this large a sample.

Recently, it has been demonstrated that the CRP/Alb ratio can be used as a biomarker for monitoring disease activity in patients with rheumatoid arthritis [17] and inflammation severity in Crohn's disease [18]. A study by
Kinoshita et al. demonstrated that the CRP/Alb ratio is a more useful prognostic factor in patients with hepatocellular carcinoma when compared to the modified Glasgow Prognostic Score [19]. The findings of the current study were different from the literature in that survival was not found to be significantly correlated with the CRP/Alb ratio among the patients with pneumonia, GI bleeding, or AKI; however, survival was significantly associated with the $\mathrm{CRP} / \mathrm{Alb}$ ratio (38 vs. 11.1, $\mathrm{p}=0.021$ ) among the patients with an acute exacerbation of CKD. For all of the patients, the APACHE II scores were strongly positively correlated with CRP ( $\mathrm{r}=0.840, \mathrm{p}<0.001)$ and CRP/Alb $(\mathrm{r}=0.852, \mathrm{p}<0.001)$. The main purpose of the current study was to investigate the relationship between the APACHE II scores and $\mathrm{CRP} /$ Alb ratio, NLR, and PLR parameters. Accordingly, the APACHE II scores were found to be strongly positively correlated with the CRP/Alb ratio, moderately positively correlated with the NLR, and weakly positively correlated with the PLR.

A retrospective study of 117 emergency ICU patients by Wang et al. reported that the RDW was an independent predictor of in-hospital mortality among elderly sepsis patients, and a high RDW was associated with poor prognosis [20]. This finding suggested that the RDW may serve as a prognostic biomarker. Sunil et al. analyzed the correlation between the RDW and other prognostic models, and 30-day mortality among adult ICU patients with AKI requiring dialysis. They found the RDW to be strongly and significantly correlated with the APACHE II scores and concluded that the RDW was a better predictor of mortality among ICU patients with AKI requiring dialysis when compared to the other disease severity scoring systems [21]. 
In contrast, RDW was not found to be significantly associated with survival herein, but a strong positive correlation between the RDW and the APACHE II scores was found $(\mathrm{r}=0.712, \mathrm{p}<0.001)$. Similarly, Zhang et al. reported a significant positive correlation between the APACHE II scores and RDW among 42 severely acute pancreatitis patients in the ICU [22]. However, there are no other studies investigating the relationship between the RDW and APACHE II scores among this many subjects.

Önal et al. investigated Alb levels and prognosis in 113 patients aged over 65 years who were admitted to the anesthesia ICU and found that Alb was significantly lower in patients who died than in those who survived $(p<0.001)$, and Alb was associated with the APACHE II scores and mortality [23]. A strong negative correlation was found in the current study between the APACHE II scores and Alb $(\mathrm{r}=-0.756, \mathrm{p}<0.001)$, but not between Alb and mortality $(\mathrm{p}=0.843)$. This discrepancy can be ascribed to the differences in the populations of the study, where all patients $\geq 18$ years of age we included in the current study, while Önal et al. included only patients $>65$ years of age. Moreover, patients receiving exogenous Alb therapy were also excluded in the current study. Mei Yin et al. evaluated serum Alb levels and sepsis prognosis among 116 ICU patients and reported the overall 28-day mortality to be $26.7 \%$, and the most common infection source to be the lower respiratory tract. Patients who died had lower serum Alb levels and higher APACHE II scores when compared to the patients who survived. Multivariate Cox regression analysis revealed the serum Alb level $(<29.2 \mathrm{~g} / \mathrm{L})$ and APACHE II score to be independent risk factors for mortality [24].

The limitations of the current study included its singlecenter design and only having evaluated the APACHE II for ICU scoring. The strengths of the study were its prospective design, large sample size, and having compared the APACHE II scores and mortality with both the biochemical and hematological parameters.

\section{Conclusions}

The APACHE II score was significantly correlated with the $\mathrm{CRP} / \mathrm{Alb}$ ratio, NLR, and PLR. The NLR and CRP/Alb were statistically associated with mortality in patients hospitalized for an acute exacerbation of CKD.

Research funding: None declared.

Author contributions: All authors have accepted responsibility for the entire content of this manuscript and approved its submission.
Competing interests: Authors state no conflict of interest. Informed consent: Informed consent was obtained from all individuals included in this study.

Ethical approval: Research involving human subjects complied with all relevant national regulations, institutional policies and is in accordance with the tenets of the Helsinki Declaration (as revised in 2013), and has been approved by the authors' institutional review board (Yildirim Beyazit University, Faculty of Medicine, Ethics Committee) or equivalent committee (Approval Number 26379996/106, Date 18/09/2019).

\section{References}

1. Bouch DC, Thompson JP. Severity scoring systems in the critical ill. Cont Educ Anesth Crit Care 2008;8:181-5.

2. Chow 0 , Barbul A. Immunonutrition: role in wound healing and tissue regeneration. Adv Wound Care 2014;3:46-53.

3. Gabay C, Kushner I. Acute-phase proteins and other systemic responses to inflammation. N Engl J Med 1999;340:448.

4. Ablij H, Meinders A. C -reactive protein: history and revival. Eur J Intern Med 2002;13:412-22.

5. Moman RN, Gupta N, Varacallo M. Physiology, albumin. In: StatPearls [Internet]. Treasure Island, FL: StatPearls Publishing; 2021. Available from: https://www.ncbi.nlm.nih.gov/books/ NBK459198/.

6. Devran O, Karakurt Z, Adıgüzel N, Güngör G, Moçin OY, Balcı MK, et al. C-reactive protein as a predictor of mortality in patients affected with severe sepsis in intensive care unit. Multidiscip Respir Med 2012;7:47.

7. Coimbra S, Oliveira H, Reis F, Belo L, Rocha S, Quintanilha A, et al. $\mathrm{C}$-reactive protein and leucocyte activation in psoriasis vulgaris according to severity and therapy. J Eur Acad Dermatol Venereol 2010;24:789-96.

8. Kasperska-Zajac A, Brzoza Z, Rogala B. Platelet function in cutaneous diseases. Platelets 2008;19:317-21.

9. Gilani MT, Razavi M, Azad AM. A comparison of simplified acute physiology score II, acute physiology and chronic health evaluation II and acute physiology and chronic health evaluation III scoring system in predicting mortality and length of stay at surgical intensive care unit. Niger Med J 2014;55: 144-7.

10. Kwon HC, Kim SH, Oh SY, Lee S, Lee JH, Choi HJ, et al. Clinical significance of preoperative neutrophil-lymphocyte versus platelet-lymphocyte ratio in patients with operable colorectal cancer. Biomarkers 2012;17:216-22.

11. Xue TC, Jia QA, Ge NL, Zhang BH, Wang YH, Ren ZG, et al. The platelet-to-lymphocyte ratio predicts poor survival in patients with huge hepatocellular carcinoma that received transarterial chemoembolization. Tumour Biol 2015;36: 6045-51.

12. Zheng J, Cai J, Li H, Zeng K, He L, Fu H, et al. Neutrophil to lymphocyte ratio and platelet to lymphocyte ratio as prognostic predictors for hepatocellular carcinoma patients with various treatments: a meta-analysis and systematic review. Cell Physiol Biochem 2017;44:967-81. 
13. Soylu K, Gedikli Ö, Ekşi A, Avcıoğlu Y, Soylu Ai, Yüksel S, et al. Neutrophil-to-lymphocyte ratio for the assessment of hospital mortality in patients with acute pulmonary embolism. Arch Med Sci 2016;12:95-100.

14. Mühl D, Füredi R, Cristofari J, Ghosh S, Bogár L, Borsiczki B, et al. Evaluation of oxidative stress in the thrombolysis of pulmonary embolism. J Thromb Thrombolysis 2006;22: 221-8.

15. Punda A, Polic S, Rumboldt Z, Bagatin J, Markovic V, Lukin A. Effects of atenolol and propranolol on platelet aggregation in moderate essential hypertension: randomized crossover trial. Croat Med J 2005;46:219-24.

16. Velissaris D, Pantzaris ND, Bountouris P, Gogos C. Correlation between neutrophil-to-lymphocyte ratio and severity scores in septic patients upon hospital admission. A series of 50 patients. Rom J Intern Med 2018;56:153-7.

17. Yang WM, Zhang WH, Ying HQ, Xu YM, Zhang J, Min QH, et al. Two new inflammatory markers associated with disease activity score-28 in patients with rheumatoid arthritis: albumin to fibrinogen ratio and C-reactive protein to albumin ratio. Int Immunopharm 2018;62:293-8.

18. Qin G, Tu J, Liu L, Luo L, Wu J, Tao L, et al. Serum albumin and C-reactive protein/albumin ratio are useful biomarkers of Crohn's disease activity. Med Sci Mon Int Med J Exp Clin Res 2016;22: 4393-400.
19. Kinoshita A, Onoda H, Imai N, Iwaku A, Oishi M, Tanaka K, et al. The $C$-reactive protein/albumin ratio, a novel inflammationbased prognostic score, predicts outcomes in patients with hepatocellular carcinoma. Ann Surg Oncol 2015;22:803-10.

20. Wang AY, Ma HP, Kao WF, Tsai SH, Chang CK. Red blood cell distribution width is associated with mortality in elderly patients with sepsis. Am J Emerg Med 2018;36:949-53.

21. Nanjarapalle S, Samantaray A, Vishnubhotla S. Red cell distribution width as a severity marker on the outcome of patients with acute kidney injury on renal replacement therapy. Indian J Crit Care Med 2020;24:95-8.

22. Zhang FX, Li ZL, Zhang ZD, Ma XC. Prognostic value of red blood cell distribution width for severe acute pancreatitis. World J Gastroenterol 2019;25:4739-48.

23. Onal O, Ozgun G. Comparison of the course and prognosis of geriatric patients admitted to the intensive care unit according to BMI and albumin values. Anesthesiol Pain Med 2016;6:e32509.

24. Yin M, Si L, Qin W, Li C, Zhang J, Yang H, et al. Predictive value of serum albumin level for the prognosis of severe sepsis without exogenous human albumin administration: a prospective cohort study. J Intensive Care Med 2018;33:687-94.

Supplementary Material: The online version of this article offers supplementary material (https://doi.org/10.1515/labmed-2021-0061). 